\title{
Tangence
}

\section{Le Livre ouvrait notre espace... petite avant-pensée crépusculaire}

Jean-Pierre Vidal

Numéro 54, mars 1997

Poétique du Livre

URI : https://id.erudit.org/iderudit/025940ar

DOI : https://doi.org/10.7202/025940ar

Aller au sommaire du numéro

Éditeur(s)

Tangence

ISSN

0226-9554 (imprimé)

1710-0305 (numérique)

Découvrir la revue

Citer cet article

Vidal, J.-P. (1997). Le Livre ouvrait notre espace... petite avant-pensée crépusculaire. Tangence, (54), 120-126. https://doi.org/10.7202/025940ar d'utilisation que vous pouvez consulter en ligne.

https://apropos.erudit.org/fr/usagers/politique-dutilisation/ 


\section{Le Livre ouvrait notre espace... petite avant- pensée crépusculaire}

Jean-Pierre Vidal

Une humanité qui a appris à marcher avec des chaussures n'a pas développé sa pensée de la même manière que si elle avait marché pieds nus.

Umberto Eco, La guerre du faux

Sans doute l'espace du livre s'est-il véritablement ouvert en Occident vers le milieu du cinquième siècle avant Jésus-Christ, au moment où, les spécialistes nous l'apprennent, la lecture, jusqu'alors toujours proférée et publique, devient silencieuse et privée. Cette voix dans la tête, qui n'en est pas une, mais qui, de nos jours, rend à certains, dont je suis, insupportable la lecture à haute voix de textes aimés - de cet amour, aux confins de la manie, qui les fait "pratiquer" comme en cette rumination que Nietzsche demandait à la lecture - parce qu'il s'agit toujours alors d'une voix "autre "et même de la voix de l'Autre, inscrite dans le monde et à la limite faisant le monde, comme on fait le mort; cette voix sourde et presque insue, au bord toujours de l'évanescence, c'est elle qui à chacun fait méconnaître sa voix "réelle", sa voix sociale, depuis que la technique nous a permis d'entendre les enregistrements de ce soma; cette voix enfin qui est la lecture dans ses premiers instants où elle tutoie l'être, c'est elle sans doute que Socrate nomme son * daimon.

On a semblablement relevé que l'intériorisation de la justice en "diké", c'est-à-dire exigence morale individuelle, est probablement liée à l'existence du thêâtre et à ce dispositif optique qui exige du citoyen pris dans la géométrie de la représentation qu'il se "situe", se purgeant ainsi mais de faire, tel un navigateur, le point. C'est en effet surtout cela la *catharsis * aristotélicienne que l'on relise la Poétique -, un rapport de triangulation qui permet au spectateur de déterminer son propre lieu depuis les deux extrêmes que sont le héros tragique et le héros comique, entre la crainte et la pitié qu'ils inspirent. Surtout, l'introduction de l'écri- 
ture, en Grèce, a coïncidé avec la naissance du sujet politique, les lois écrites sur les parois du Prytanée, les lois murs, étant en quelque sorte la matérialisation, en forme de livre, de l'inscription de la cité dans le monde, du passage de la horde sans attache au retrait dans un territoire qui est, bien entendu, mental avant tout. Et l'édifice tourne ses murs de lois vers son dehors, afin que nul n'en ignore, tout autant qu'il les fait servir à cerner ce dedans virtuel que représente, pour chacun des citoyens, la loi, dans laquelle siègent ainsi les magistrats. Sensiblement au même * moment "de la pensée grecque, CEdipe, sujet par nature mal placé, en trop, devient, s'il faut en croire Jean-Joseph Goux ${ }^{1}$, la figure mythique du philosophe, dans la mesure où la réponse qu'il donne à l'énigme de la Sphinge est le fruit d'une réflexion, d'une déduction logique plutôt que d'une leçon apprise et parce que la sagesse qu'au-delà de ses drames il finit par atteindre s'avère le résultat d'une maturation individuelle, de l'expérience, de son expérience de "marcheur" de monde et non plus celle de la tradition transmise, du collectif qui, mortellement pour le sujet, voyait toujours à tout. Devant l'énigme du monde, CEdipe est seul enfin, comme tout lecteur. Seul grâce à l'Autre qui l'a sommé à comparaître. L'Autre dans son cas, comme dans celui de Thésée, comme dans celui de la plupart des héros grecs qui disent la conquête concomitante du politique et de l'éthique, de l'espace public et de l'espace privé, l'Autre c'est le chaos intenable des formes sans raison, de la germination sans ordre, de l'hybride et du monstre qu'est toujours le demi-humain, l'humain à peine, l'humain pas encore, l'humain inabouti. Ou, comme il semble que nous le vivions désormais, de l'humain dépassé.

Nous devons, je crois, au livre, théâtre interpellant par l'ouverture de la scène et l'appel d'air de la salle, édifice légal qui dit expressément, objectivement, qu'il y a de l'implicite, nous devons au livre un rapport à l'Autre qui éventre littéralement notre espace, qui l'invagine, le retourne et surtout l'écartèle en un carrefour de forces centripètes et centrifuges, entre l'impossible unité du sujet et l'impensable multiplicité du monde. Le livre est cette cloison qui fait sas, ce miroir qui ne répercute que d'être traversé, cet édifice où l'on ne peut entrer sans en être aussitôt sorti. Le livre est la coupure rendue visible et comme telle aussitôt suturée.

1 Voir son Edipe philosophe, Paris, Aubier, coll "La psychanalyse prise au mot ", Paris, 1990, 222 p. 
Et peut-être l'espace du livre est-il en train de se refermer, en Occident, avec la disparition, partout attestée, du sujet politique mais aussi esthétique et éthique, dans cet oxymoron pétrifiant et pétrifié : le sujet de masse, le sujet de consommation dont le signe est à la fois instantané et virtuel, c'est-à-dire qu'au lieu de la dire, de l'arpenter et ainsi, dans la mesure de ses moyens, de la maîtriser, il rend folle la distance. En la comblant, en l'évacuant pour en faire l'impensé qui fait défaillir le sujet. Ainsi la Sphinge techniciste et "à l'écoute des faits», des lois du marché, de l'état des choses, de tout ce qui représente le tautologique sur quoi l'on veut refermer l'homme, cette ouverture, ainsi la Sphinge a-t-elle eu raison d'CEdipe et de ses exigences de devenir et d'incertain fondateur ${ }^{2}$; ainsi le Minotaure (dont, bien plus que du labyrinthe, Internet pourrait bien être une menaçante métaphore) a-t-il fini par dévorer Thésée.

La déterritorialisation monétaire et économique, cette libération extravagante du capital - un capital qui est anonymat absolu et abstraction totale - devenu le grand principe monstrueux de la fin du siècle, produit, on le sait, une désolidarisation dont les effets se font sentir dans tous les modes du social, et en particulier dans l'éducation comme dans la culture. À un autre niveau, elle produit une "désolidarisation" de l'œuvre d'art dans la méconnaissance ou le refus de considérer qu'elle est un espace ouvertement clos (solidaire au sens étymologique, c'est-à-clire en volume, en relief. Et ce volume s'appelle le sujet) au profit d'une perverse excitation brownienne de particules agitées et de petits " moi " frivoles campés sur leur quant-à-soi immobile et dérisoire.

Ce qui s'oublie ainsi c'est le signe, ramené à une émulsion ou, pire encore, une démangeaison ponctuelle comme un pixel. Car le fondement du signe est une relation d'altérité et non de

2 Il y a, en effet, dans la réponse d'Oedipe, à la fois les conditions d'une généralité triomphante: l'homme et une implicite acceptation, en forme de réponse à l'appel, en forme de "présent ", l'acceptation d'être pris soi-même, en tant que sujet, dans la mineure d'un syllogisme qui est proprement le rapport d'altérité fait discours: la réponse est l'Homme, or je suis un homme. C'est par cela. par cela seulement, que l'on chasse les monstres. Mais cela est un incertain fondateur dans la mesure où cela se doit, pour qu'il y ait sujet, de rester une question perpétuellement reconduite, sans cesse à trancher ("Toute réponse ne garde sa force de réponse qu'aussi longtemps qu'elle reste enracinée dans le questionnement., Heidegger). Et cela, en fin de compte, s'appelle l'éthique. 
commodité ou d'adéquation comme le postulent la philosophie anglo-saxonne du langage et ses avatars contemporains qui assimilent l'art à la communication et font de la lecture le producteur de l'ouvre. Une relation d'altérité, c'est-à-dire de négativité, c'està-dire encore d'opposition, de complémentarité et d'abstraction, mais sans qu'aucune de ces trois forces ne se puisse concevoir indépendamment des deux autres. Le signe implique espacement et disposition, à l'intérieur du sujet comme à l'intérieur du monde, et de l'un à l'autre, de l'un vers l'autre. Ce signe, au lieu de le laisser sans contour, diffus, invisible dans la langue, pneumatique et nébuleux, le livre, et plus généralement le lieu de l'œuvre d'art, en délimitent l'espacement, lui donnent une "portée", c'est-à-dire à la fois une architecture de présentoir et d'arpentage sur quoi s'inscrire et une "allonge " vers le sujet et vers le monde. Comme le rythme, certes antérieur à la mesure, ne se rend vraiment visible et surtout germinant qu'à l'inscription sur la portée, le signe, ce rythme de l'inscription dans le monde, cette ponctuation du monde et du social dans et par le sujet, ne se déploie dans toute son envergure "pensante " et "ouvrante", comme disait Heidegger du langage (dans son séminaire sur Héraclite), que dans l'espace de l'ouuvre d'art parce qu'il y est à la fois "jeu", dans le double sens d'activité libre et joyeuse et de passivité contrainte et clivée, passion donc, et "travail ", dans le double sens d'activité et de passivité encore, d'exercice d'une force sur une matière ou dans un espace et d'animation d'une matière ou d'un espace par une force cette fois a interne". Et c'est, pour moi, le livre et la lecture silencieuse qui ouvrent à l'écriture l'espace de l'art, loin de la publicité assourdissante de la voix, trop immédiate et d'entrée collective mais de droit divin, d'emblée civique mais par inadvertance.

L'écriture dans le livre, dans le silence et dans la tête, résonne comme un appel. Et parce qu'elle est, dans cet espace au moins double (la page et ce de moi à quoi elle aboutit et qui la re-suscite), non seulement le signe d'une distance mais la distance, la distance rendue visible, cet appel, cette sommation ne sont pas le réconfort social d'un présent, d'une assemblée, d'une coïncidence ou, à tout le moins, d'une convergence, mais la violence, anarchique et civique à la fois, d'un futur qui ne s'ouvre qu'alors, au moment de cette stase imposée au monde et au sujet.

"Le monde est dans ma tête. Mon corps est dans le monde ", dit admirablement Paul Auster, dans une simplicité qui résonne 
longtemps, comme un oracle. Car elle montre très clairement que le sujet contient ce qui le contient, que le monde n'est tel que lorsqu'il est pensé et que cette pensée est "au monde", c'est-àdire non seulement qu'elle lui est adressée mais que l'effet de cette adresse et cette adresse elle-même adviennent "réellement ". C'est l'autre sens, occulté, de la mimèsis grecque, celle d'Aristote dont Philippe Lacoue-Labarthe a montré, reprenant un texte de la Pbysique qu'elle fondait toute sa théorie du théâtre et donc de l'œuvre d'art:

Aristote dit tout d'abord (194a) qu'en général *l'art imite la nature : bè téchnè mimeitai tèn pbusin. Puis, un peu plus loin (199a), il spécifie cette relation générale de mimèsis: d'une part, dit-il, la téchnè mène à son terme (accomplit, perfectionne, épitélet) ce que la pbusis est incapable d'outvrer (apergasasthai); d'autre part, elle imite.

Il y a donc deux mimésis (je passe du grec au français) : une mimésis restreinte, qui est la reproduction, la copie, la réduplication de ce qui est donné - déjà cuuvré, effectué, présenté par la nature $[\ldots]$.

Et puis il y a une mimésis générale, qui ne reproduit rien de donné (qui ne re-proxluit donc rien du tout) mais qui supplée à un certain défaut de la nature, à son incapacité à tout faire, tout ceuvrer, tout produire. C'est une mimésis productive, c'està-dire une imitation de la phusis comme force productrice ou, si l'on prëfère, comme poüesis. ${ }^{3}$

C'est aussi ce que dit, hors le discours et la pratique psychanalytiques où cette figure reçoit bien sûr d'autres effets - mais cette "sortie" du contexte s'autorise du fait que la théorie du fondateur de l'école freudienne de Paris est aussi une sémiotique le $Z$ lacanien. Car le stade du miroir, comme la mimésis bifide d'Aristote, ouvre un espace de répercussions, du monde au sujet et retour, un espace où l'un désormais ne va pas sans l'autre, mieux, où l'un prolonge l'autre, y œuvre et s'en trouve modifié. À l'horizon de cet espace se lève le livre de Mallarmé comme le monument de la suppléance.

Le sujet est (une) fonction du monde, tel est, à mon sens, le message fondamental de l'ouvre d'art. Et c'est ainsi que l'oeuvre elle-même est le "devenir-Signe" de l'artiste. Elle est le simulacre

3 L'imitation des modernes, Paris, Galilée, coll. * La philosophie en effet *, 1986, 289 p. 
d'une présence qui n'est plus celle de son producteur - et encore moins celle de ce que la vulgate communicationnelle qui nous contraint au médiatique nomme son "récepteur" - mais le simulacre de la présence, de la présence, mettons de l'humain, et de l'"apparaître du monde, en tant que le geste mimétique les convoque et les conjoint. Un simulacre qui prend la forme d'un désir, d'une intentionnalité dont il ne reste que la trace, comme un remords, ou plutôt comme le signe d'un retrait.

Sur la scène de l'ouvre, dans l'ouverture du livre, le rapport à l'Autre qui englobe à la fois l'objectivation de la présence, la chosification dans l'ouvre et le rapport à autrui, à travers cette chosification, au-delà d'elle mais à partir d'elle, le rapport à l'Autre est une ouverture à l'Être. Car dans cette triangulation qui, de part et d'autre de l'ouvre, unit deux sujets à la chose, à l'événement, à l'espace qu'elle est, et au-delà à la Chose et à l'Événement, le rapport primordial est du sujet (que celui-ci soit le producteur de l'œuvre ou celui qui la reçoit ou plutôt la reconcluit) à la chose et non d'un sujet à l'autre. Et le corollaire de cet axiome c'est que l'œuure, le livre, représente la substitution réglée d'un sujet à un autre. Car l'CEuvre est "du "sujet toujours en émergence, l'CEuvre est, thêâtralement, le Sujet même, mais toujours en instance.

Nous rencontrons ici la pensée de Heidegger qui dans "l'origine de l'œuvre d'art " ${ }^{4}$ fonde le sens de l'wuvre d'art sur le rapport de l'CEuvre à la Chose en soi d'une part, et sur le rapport de l'CEuvre à l'Art d'autre part, et partant (et partant seulement) à l'homme. Car l'homme, seul "étant" dont l'existence à la fois questionne le sens de l'Être et est faite de ce questionnement même, de l'entendement qu'il a de son sens, l'homme est toujours en porte-à-faux, toujours en différé, parce que toujours déjà "jeté" dans le monde (c'est aussi, fondamentalement, la leçon de Lacan) et que l'CEuvre, *acheminement " vers l'Être, "ouverture" à l'Être, *illumination" de l'Être, tous termes par lesquels Heidegger désigne ce qu'en termes plus prosaïques et certes réducteurs on pourrait définir comme le rapport au sens, l'CEuvre donc est l'une des rares instances, l'un des "sentiments" clit Heidegger, qui permet à l'homme de se saisir comme ce porte-à-faux, ce différé, ce

4 Dans Chemins qui ne mènent nulle part, laris, Gallimard, coll. •Tel *, 1986 (1949 pour l'original allemand, 1962 pour la traduction française). 461 p. 
déjà-là à-venir qu'il est. Et ainsi de se donner forme, de se voir projet et de se perdre chose.

À la perverse polymorphie d'un sujet qui s'éclate, se sait éclaté dans la matière des cuvres et les contours qu'il leur donne répond la moire anamorphique de l'œeuvre qui s'inscrit dans le monde de telle façon que de ses plis elle en défait la trame et la refait autre, entraînant dans ce balayage le sujet et son rapport constitutif à l'Autre en même temps qu'elle fait de l'apparition de l'Autre sur sa scène et dans son cérémonial une convocation du sujet, toujours dès lors en position stroboscopique d'intermittence et d'altération.

Mais pour ceci il nous fallait une scène, il nous fallait un seuil, il nous fallait le livre. Aujourd'hui que nous portons sur le monde le regard de la mouche, prismatique, émietté, multipliant, pléthorique, désorienté, notre esthétique est celle du bariolage et de la juxtaposition sans calcul, c'est-à-dire de l'absence de dégradé, de progressivité par quoi l'un devient l'autre, ou même au contraire l'absence de résistance par quoi l'un provient de l'autre dans l'opposition et la projection, bref l'absence de seuil ou de sas qui clise l'importance de la distance clans le départage de l'humain. De plein pied dans le collage, nous avons la faiblesse théorique de prétendre voir un métissage dans ce qui n'est qu'un confortable babélisme des quant-à-soi adverses et irréconciliés.

La scène, le livre se sont effrités en écrans où à défaut d'Autre scintillent et passent, comme de gentils électrons, dinsignifiants petits autres qui nous rapetissent à leur image de lucioles corpusculaires.

Un grand manque de Sujet est en train de nous trover l'âme. 